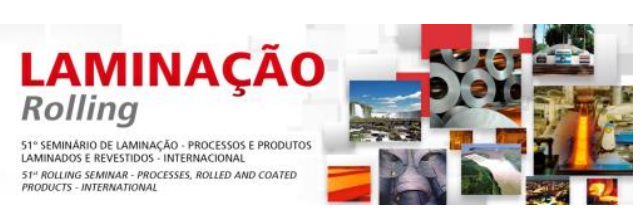

\title{
SIMULAÇÃO POR ELEMENTOS FINITOS DO PROCESSO DE LAMINAÇÃO DE TIRAS A QUENTE DE AÇOS C-Mn*
}

\author{
Gustavo Coqui Barbosa' \\ Luciano Pessanha Moreira²
}

\section{Resumo}

Neste trabalho, foi desenvolvido um modelo de elementos finitos para simular o primeiro passe da laminação a quente do trem acabador de tiras de aço C-Mn. O comportamento desse aço é definido por equações constitutivas que descrevem os mecanismos de encruamento e recristalização acoplados aos campos de tensões, deformações e temperatura. Foi implementada uma sub-rotina em linguagem FORTRAN disponível no programa de elementos finitos ABAQUS/Standard. O modelo foi estabelecido no estado plano de deformação por meio de elementos finitos com acoplamento dos campos de temperatura e deformação para o cilindro de trabalho e tira. As dimensões e condições de processo foram obtidas a partir de dados industriais do laminador de tiras a quente da CSN. O modelo prevê as distribuições de temperaturas e valor de carga de laminação em boa concordância com as medidas industriais. Os resultados do modelo evidenciam que há ocorrência de recristalização dinâmica durante 0 primeiro passe $e$, apontam que em determinadas regiões da tira a fração recristalizada dinamicamente chega a $100 \%$. A análise de sensibilidade dos parâmetros do modelo foi feita para determinar o seu impacto nos resultados das simulações, notadamente na força de laminação e no tamanho de grão austenítico final.

Palavras-chave: Laminação a quente; Método de elementos finitos; Aço C-Mn.

\section{FINITE ELEMENT SIMULATION OF THE HOT STRIP ROLLING PROCESS OF C-Mn STEELS}

\begin{abstract}
In this work, a finite element model was developed to simulate the first pass of finishing hot rolling mill of C-Mn steel strips. The behavior of this steel is defined by constitutive equations that describe the mechanisms of hardening and recrystallization coupled to the stress, strain and temperature fields. A subroutine was implemented in FORTRAN available in the finite element ABAQUS/Standard commercial code. The model was established assuming the plane strain state by means of thermomechanical coupling elements for the work roll and the steel strip. The process conditions were obtained from industrial data of the CSN's hot rolling mill. The model predicts the distribution of temperatures and rolling load value in good agreement with industrial measurements. The model results show that there is occurrence of dynamic recrystallization during the first pass, and revealed that in certain regions of the strip dynamically recrystallized fraction reaches $100 \%$. The sensitivity analysis of the model parameters was performed to determine their impact on the simulation results, namely the rolling load and the austenitic grain size.
\end{abstract}

Keywords: Hot rolling; Finite element method; C-Mn steel.

1 Doutor, Professor Associado do Departamento de Engenharia Mecânica, Escola de Engenharia Industrial Metalúrgica de Volta Redonda - UFF, Volta Redonda, Rio de Janeiro, Brasil.

2 Mestre, Programa de Pós-Graduação em Engenharia Metalúrgica, Escola de Engenharia Industrial Metalúrgica de Volta Redonda - UFF, Volta Redonda, Rio de Janeiro, Brasil.

* Contribuição técnica ao $51^{\circ}$ Seminário de Laminação - Processos e Produtos Laminados e Revestidos, 28 a 31 de outubro de 2014, Foz do Iguaçu, PR, Brasil. 


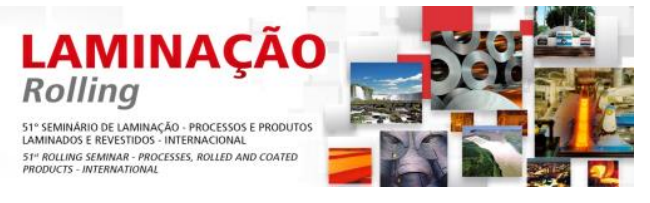

Tabela 1. Parâmetros do processo de laminação quente para o primeiro passe [1].

\begin{tabular}{c|c|c|c|c|c|c}
\hline $\begin{array}{c}\text { Espessura } \\
\text { de } \\
\text { entrada }\end{array}$ & $\begin{array}{c}\text { Espessura } \\
\text { de saída }\end{array}$ & Redução & $\begin{array}{c}\text { Largura da } \\
\text { tira }\end{array}$ & $\begin{array}{c}\text { Raio do } \\
\text { cilindro }\end{array}$ & $\begin{array}{c}\text { Temperatura } \\
\text { da tira }\end{array}$ & $\begin{array}{c}\text { Rotação do } \\
\text { cilindro }\end{array}$ \\
\hline $35,40 \mathrm{~mm}$ & $24,8 \mathrm{~mm}$ & $30 \%$ & $1.200 \mathrm{~mm}$ & $366 \mathrm{~mm}$ & $984,7^{\circ} \mathrm{C}$ & $37 \mathrm{rpm}$ \\
\hline
\end{tabular}

\subsection{Modelamento do Processo de Laminação a Quente}

\subsubsection{Equações Constitutivas}

O comportamento típico de um aço baixo carbono deformado em altas temperaturas como, a título de exemplo, no processo de laminação a quente de aços, é apresentado na Figura 1. Os mecanismos de restauração são respectivamente denotados por dinâmico e estático dependendo se estes ocorrem concomitantemente com a aplicação da tensão ou não. No início do processo de deformação, o material sofre encruamento devido ao aumento da densidade de discordâncias as quais se arranjam em contornos de subgrãos. À medida que o nível de deformação plástica aumenta, tem-se que a aniquilação de discordâncias e geração de novas discordâncias geram, como resultado, um estado estacionário na curva tensão de escoamento-deformação mostrado no platô (a) da Figura 1. Tal mecanismo é conhecido como recuperação dinâmica cuja microestrutura resultante é formada por subgrãos bem definidos que, por sua vez, são a fonte para a nucleação da recristalização estática [1] ${ }^{1}$.

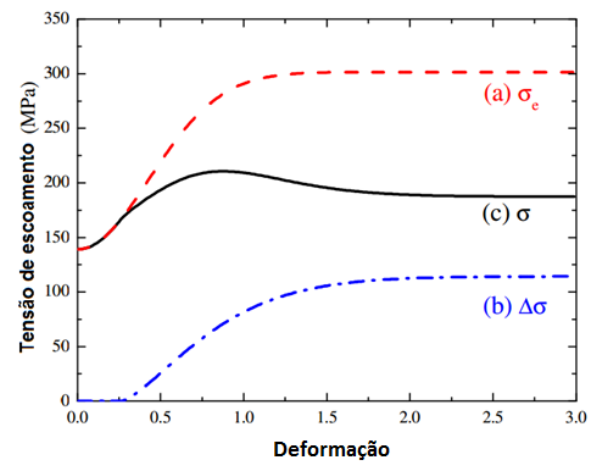

Figura 1. Curva tensão de escoamento-deformação para um aço baixo carbono determinada para um tamanho de grão inicial de $20 \mu \mathrm{m}$ e valores constantes de taxa de deformação $\left(4,5 \mathrm{~s}^{-1)}\right.$ e temperatura de $900^{\circ} \mathrm{C}$ descrevendo os mecanismos de: (a) recuperação dinâmica; (b) amaciamento devido a recristalização (c) a superposição dos eventos (a) e (b) [1].

Quando a recuperação dinâmica é o único processo de restauração ativo a tensão de escoamento pode ser descrita como [2-4]:

$\sigma_{e}=\sigma_{0}+\left(\sigma_{S S}-\sigma_{0}\right)[1-\exp (-C \varepsilon)]^{m}$

na qual $\sigma_{0}$ é a tensão de início de escoamento plástico, $\sigma_{S S}$ é a tensão de escoamento para o estado estacionário e $m$ é o expoente de encruamento. A constante $\mathrm{C}$ é determinada por [2-4]:

$C=10\left[\left(\sigma_{01}-\sigma_{0}\right) /\left(\sigma_{S S}-\sigma_{0}\right)\right]^{2}$

e depende do parâmetro de Zener-Hollomon (Z) [2-4]:

$\mathrm{Z}=\dot{\bar{\varepsilon}} \exp [Q /(R T)]$

onde $\dot{\bar{\varepsilon}}$ é a taxa de deformação efetiva, $R$ é a constante dos gases $(8,314 \mathrm{~J} / \mathrm{K}) T$ é a temperatura absoluta de deformação e $Q$ é a energia de ativação para a deformação que varia entre $262-366 \mathrm{KJ} / \mathrm{mol}$ para aços C-Mn [5,6]. Da mesma forma, as tensões

\footnotetext{
* Contribuição técnica ao $51^{\circ}$ Seminário de Laminação - Processos e Produtos Laminados e
} Revestidos, 28 a 31 de outubro de 2014, Foz do Iguaçu, PR, Brasil. 
$\sigma_{0}, \sigma_{S S}, \sigma_{01}, \sigma_{S S}$ dependem da condição de deformação plástica e podem ser definidas por [2-4]:

$\sigma_{\mathrm{TE}}=\mathrm{A}_{1} \sinh ^{-1}\left(\frac{\mathrm{Z}}{\mathrm{A}_{2}}\right)^{\mathrm{A}_{3}}$

na qual $\mathrm{A}_{n}$ são parâmetros do material determinados a partir de dados experimentais, por exemplo, por compressão plana a quente ou ensaio de torção. A Tabela 2 lista os parâmetros que descrevem o comportamento de um aço C-Mn durante a laminação de tiras a quente.

Tabela 2. Parâmetros materiais do aço C-Mn [3].

\begin{tabular}{c|c|c|c|c|c|c|c|c}
\hline \multicolumn{2}{c|}{$\sigma_{0}$} & \multicolumn{5}{c}{$\sigma_{\text {ss }}$} & \multicolumn{3}{c}{$\sigma_{01}$} \\
\hline $\mathrm{A}_{1}$ & $\mathrm{~A}_{2}$ & $\mathrm{~A}_{3}$ & $\mathrm{~A}_{4}$ & $\mathrm{~A}_{5}$ & $\mathrm{~A}_{6}$ & $\mathrm{~A}_{7}$ & $\mathrm{~A}_{8}$ & $\mathrm{~A}_{9}$ \\
\hline 103,84 & $4,92 \times 10^{13}$ & 0,13 & 103,41 & $1,77 \times 10^{11}$ & 0,217 & 89,29 & $2,55 \times 10^{11}$ & 0,182 \\
\hline \multicolumn{5}{c|}{$\sigma_{\text {ss }}$} & \multicolumn{5}{c|}{$\Delta \sigma$} & & $\varepsilon_{\mathrm{p}}$ \\
\hline $\mathrm{A}_{10}$ & $\mathrm{~A}_{11}$ & $\mathrm{~A}_{12}$ & $\mathrm{k}^{\prime}$ & $\mathrm{m}^{\prime}$ & $\mathrm{a}$ & $\mathrm{A}$ & $\mathrm{p}$ & $\mathrm{q}$ \\
\hline 106,72 & $3,88 \times 10^{12}$ & 0,146 & 0,49 & 1,4 & 0,8 & $5,6 \times 10^{-4}$ & 0,3 & 0,17 \\
\hline
\end{tabular}

Por outro lado, quando o processo de recuperação é insuficiente para diminuir a energia de ativação da deformação, a multiplicação das discordâncias pode produzir a nucleação e o crescimento de grãos recristalizados. Neste caso, a curva de escoamento se eleva a um valor máximo, tensão de pico. O começo da recristalização dinâmica é caracterizado pela deformação crítica [2-4]:

$\varepsilon_{\mathrm{c}}=a \varepsilon_{\mathrm{p}}$

que é estimada pela deformação de pico dada por [2-4]:

$\varepsilon_{p}=\mathrm{Ad}_{0}^{\mathrm{p}} \mathrm{Z}^{q}$

onde do é tamanho de grão inicial e $A, p$ e q são parâmetros do material dados na Tabela 2. O amaciamento devido à recristalização dinâmica, Figura 1, curva (b), é dado por [2-4]:

$$
\Delta \sigma=\left(\sigma_{S S}-\sigma_{S S}^{\prime}\right)\left\{1-\exp \left[-\mathrm{k}^{\prime}\left(\frac{\bar{\varepsilon}-\mathrm{a} \varepsilon_{p}}{\varepsilon_{p}}\right)^{\mathrm{m}^{\prime}}\right]\right\}
$$

onde $\sigma_{S S}^{\prime}$ é descrito pela Equação (4) e k'e m'são parâmetros do material. A curva (c) na Figura 1 é obtida pela superposição dos efeitos da recuperação dinâmica e da recristalização dinâmica, isto é [2-4]:

Se $\bar{\varepsilon}<\varepsilon_{c} \therefore \bar{\sigma}=\sigma_{e}$

e

Se $\bar{\varepsilon} \geq \varepsilon_{c} \therefore \bar{\sigma}=\sigma_{e}-\Delta \sigma$

A fração volumétrica recristalizada estaticamente após um tempo t é descrita pela aplicação da clássica equação empírica de Johnson-Mehl-Avrami-Kolmogorov [7,8]:

$\mathrm{X}=1-\exp \left[-0,693\left(\mathrm{t} / \mathrm{t}_{0,5}\right)^{\mathrm{k}}\right]$

* Contribuição técnica ao $51^{\circ}$ Seminário de Laminação - Processos e Produtos Laminados e Revestidos, 28 a 31 de outubro de 2014, Foz do Iguaçu, PR, Brasil. 


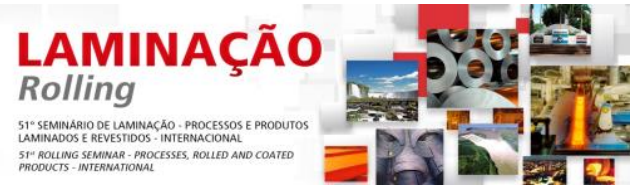

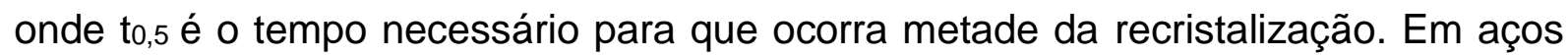
C-Mn o expoente $k$ é igual a 1,0 para recristalização estática e 1,5 para recristalização metadinâmica [9].

A recristalização dinâmica durante a laminação somente ocorrerá caso a deformação ultrapasse a deformação crítica. Para esse caso, emprega-se a Equação 4.15, que representa a fração de recristalização dinâmica do material [10-12].

$X_{\text {RexDN }}=1-\exp \left[-0,693\left(\frac{\varepsilon-\varepsilon_{c}}{\varepsilon_{0,5}}\right)^{2}\right]$

Nesta última equação, $\varepsilon 0,5$ é a deformação para $50 \%$ de recristalização dinâmica determinada por [10-12]:

$\varepsilon_{0,5}=1,144 \times 10^{-5} d_{0}^{0,28} \dot{\varepsilon}^{0,05} \exp (53388 / R T)$

As equações correspondentes ao aço C-Mn para o tempo de metade de recristalização e o tamanho de grão recristalizado $d$ para eventos de recristalização estática (RexES) e recristalização metadinâmica (RexMD) e dinâmica (RexDN) são apresentadas abaixo [9,13]:

Se $\bar{\varepsilon}<\varepsilon_{c}$

$$
\begin{aligned}
& \mathrm{t}_{0,5}^{\text {RexES }}=2,3 \times 10^{-15} \varepsilon^{-2,5} \mathrm{~d}_{0}^{2} \exp \left(\frac{230000}{\mathrm{RT}}\right) \\
& \mathrm{d}_{\text {RexES }}=343 \varepsilon^{-0,5} \mathrm{~d}_{0}^{0,4} \exp \left(-\frac{45000}{\mathrm{RT}}\right)
\end{aligned}
$$

Se $\bar{\varepsilon} \geq \varepsilon_{c}$

$$
\begin{aligned}
& \mathrm{t}_{0,5}^{\text {RexMD }}=1,1 \mathrm{Z}^{-0,8} \exp \left(\frac{230000}{\mathrm{RT}}\right) \\
& \mathrm{d}_{\text {RexMD }}=6,8 \times 10^{4} \mathrm{Z}^{-0,23}
\end{aligned}
$$

Se $\bar{\varepsilon} \geq \varepsilon_{c}$ e $\dot{\varepsilon}>0$

$$
\mathrm{d}_{\mathrm{RexD}}=3,9 \times 10^{4} \mathrm{Z}^{-0,23}
$$

\subsubsection{Condições simuladas}

Os parâmetros do modelo que foram analisados neste trabalho estão relacionados ao comportamento microestrutural do aço C-Mn: energia de ativação para deformação e tamanho de grão inicial e as condições de processo: coeficiente de troca de calor entre o cilindro e a tira e coeficiente de atrito. De acordo com a Tabela 3, foram adotados dois valores diferentes para cada parâmetro investigado, num total de 6 simulações. 


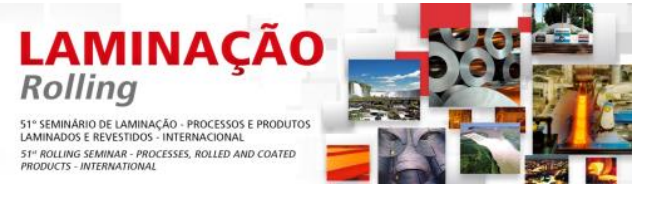

Tabela 3. Variações de parâmetros materiais e condições de processo.

\begin{tabular}{|c|c|c|c|c|c|}
\hline \multirow{2}{*}{$\begin{array}{l}\text { Parâmetro } \\
\text { Alvo }\end{array}$} & \multirow{2}{*}{ Simulação } & \multicolumn{4}{|c|}{ Dados de entrada } \\
\hline & & Q & $\mu$ & $\mathrm{TG}$ & $h_{c}$ \\
\hline \multirow{2}{*}{$\begin{array}{c}\text { Energia de ativação } \\
\text { para a deformação Q } \\
(\mathrm{J} / \mathrm{mol})\end{array}$} & 1 & 300.000 & 0,25 & 120 & 5.000 \\
\hline & 2 & 282.000 & 0,25 & 120 & 5.000 \\
\hline \multirow{2}{*}{$\begin{array}{c}\text { Coeficiente de atrito } \\
\mu\end{array}$} & 1 & 300.000 & 0,25 & 120 & 5.000 \\
\hline & 3 & 300.000 & 0,35 & 120 & 5.000 \\
\hline \multirow{2}{*}{$\begin{array}{c}\text { Tamanho de grão inicial } \\
\text { TG }(\mu \mathrm{m})\end{array}$} & 4 & 282.000 & 0,25 & 200 & 5.000 \\
\hline & 2 & 282.000 & 0,25 & 120 & 5.000 \\
\hline \multirow{2}{*}{$\begin{array}{c}\text { Coeficiente de troca de } \\
\text { calor por condução } \\
\left(\mathrm{kWm}^{-2}{ }^{\circ} \mathrm{K}^{-1}\right) \\
\mathrm{h}_{\mathrm{c}}\end{array}$} & 5 & 282.000 & 0,35 & 200 & 10.000 \\
\hline & 6 & 282.000 & 0,35 & 200 & 5.000 \\
\hline \multicolumn{2}{|c|}{ Resultados comparados } & \multicolumn{4}{|c|}{$\begin{array}{l}\text { Tamanho de grão final e } \\
\text { força de laminação. }\end{array}$} \\
\hline
\end{tabular}

\section{RESULTADOS E DISCUSSÃO}

\subsection{Previsões Numéricas}

De forma geral, as simulações numéricas apresentaram um campo de distribuição das variáveis semelhantes. Como pode ser visto na Figura 2(a), os níveis de deformação plástica equivalente mudam drasticamente na região de laminação. Além disso, vale a pena observar uma não uniformidade da deformação plástica. Isto se deve a uma repentina mudança nos campos de velocidade de laminação na direção vertical, visto que a região de mais alta taxa de deformação ocorre junto a região de entrada, e subsequentemente ela decai gradualmente ao longo do arco de contato, Figura 2(b). O gradiente de temperatura na direção da espessura é cada vez mais intenso a medida que a espessura da tira é reduzida. As principais razões para isso, podem ser atribuídas a transferência de calor devido o contato da tira com o cilindro de trabalho e a geração de calor devido a deformação plástica, Figura 2(c). $\mathrm{Na}$ Figura 2(d) pode ser visto que o grão austenítico é refinado na zona de mordida devido a recristalização dinâmica e recristalização metadinâmica [14,15].

* Contribuição técnica ao $51^{\circ}$ Seminário de Laminação - Processos e Produtos Laminados e Revestidos, 28 a 31 de outubro de 2014, Foz do Iguaçu, PR, Brasil. 


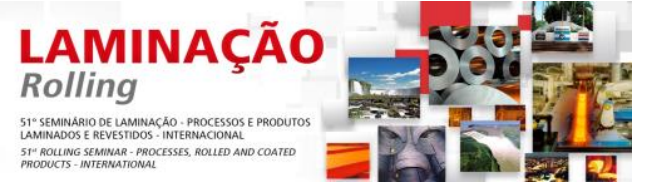

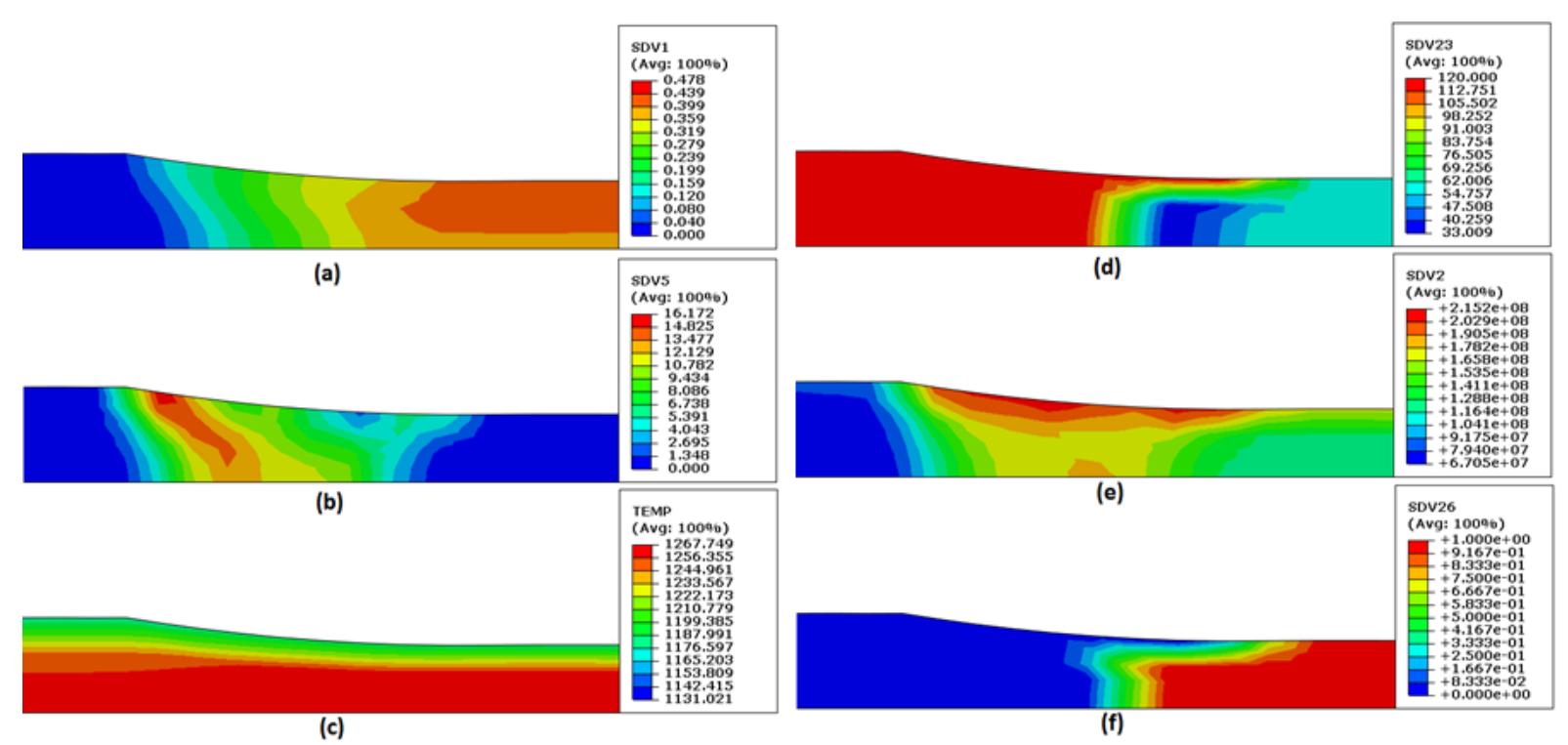

Figura 2. Campo de distribuição das variáveis no primeiro passe de laminação de tiras a quente para a simulação 1: (a) deformação plástica equivalente; (b) taxa de deformação plástica equivalente $\left(\mathrm{s}^{-1}\right)$; (c) temperatura $(\mathrm{K})$; (d) tamanho de grão $(\mu \mathrm{m}) ;(\mathrm{e})$ tensão de escoamento (Pa) e (f) fração amaciada [15].

\subsection{Análise de Sensibilidade}

A análise de sensibilidade dos parâmetros do modelo foi feita para determinar o seu impacto nos resultados das simulações, notadamente na força de laminação e no tamanho de grão austenítico final.

\subsubsection{Energia de ativação para a deformação}

Segundo Gorni [16], a energia de ativação para deformação, é um parâmetro de fundamental importância para a caracterização do comportamento de metais durante sua conformação a quente, pois indica a energia necessária para a ativação dos mecanismos de amaciamento dinâmico que reduzirão sua resistência à deformação a quente. Nos aços C-Mn, o valor dessa energia varia entre 262-366 kJ/mol. Logo, para avaliar a sensibilidade desse parâmetro nos principais resultados do primeiro passe de laminação, foram adotados os valores de $282 \mathrm{~kJ} / \mathrm{mol}$ e $300 \mathrm{~kJ} / \mathrm{mol}$. Para fins de comparação, foram escolhidas as simulações 1 e 2. Espera-se que quanto menor for energia de ativação para deformação mais atuantes serão os mecanismos de restauração, que, por consequência acarretariam em uma menor tensão de escoamento e isso se refletiria em um menor valor para a força de laminação [15].

A comparação entre as curvas de distribuição de pressão determinadas a partir das simulações 1 e 2 é apresentada na Figura 3(a). Como pode ser observado, a distribuição da pressão de contato entre o cilindro e a tira foi menor para a simulação 2 do para a simulação 1. Isso mostra que os resultados da simulação foram consistentes com o que se esperava do comportamento do aço C-Mn [15].

A força de laminação calculada para a simulação 2 foi de $14,28 \mathrm{MN}$ e a prevista para a simulação 1 foi de $18,00 \mathrm{MN}$, ou seja, para um aumento de apenas $6 \%$ na energia de ativação para a deformação houve um aumento de 20,66 \% na força de laminação calculada. Esse resultado indica claramente que energia de ativação para a deformação exerce um papel importante sobre o cálculo da força de laminação [15].

* Contribuição técnica ao $51^{\circ}$ Seminário de Laminação - Processos e Produtos Laminados e Revestidos, 28 a 31 de outubro de 2014, Foz do Iguaçu, PR, Brasil. 


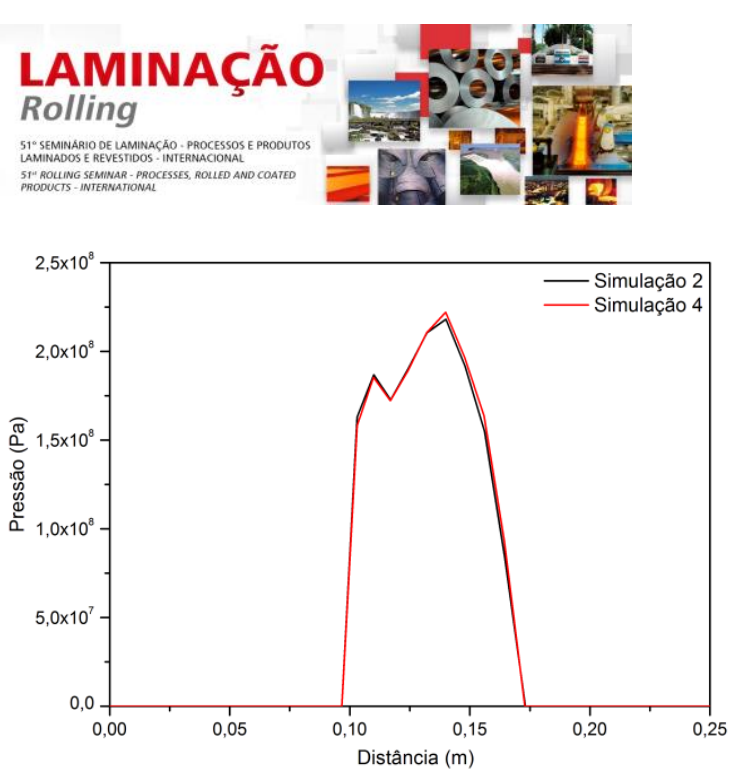

(a)

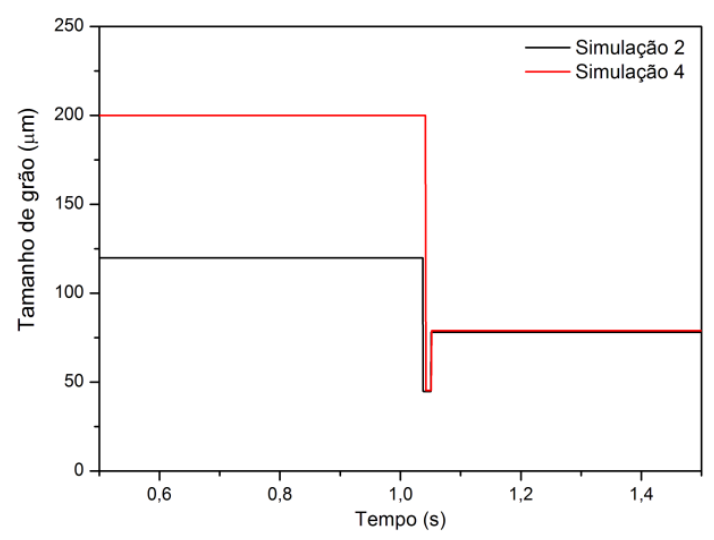

(b)

Figura 5. (a) Distribuições de pressão ao longo do arco de contato, (b) evolução do tamanho de grão austenítico [15].

\subsubsection{Coeficiente de troca de calor}

As Figuras 6(a) e 6(b) apresentam as curvas de distribuição de pressão e evolução do tamanho de grão austenítico, respectivamente. Nota-se claramente que não houve mudança na posição dessas curvas, apesar de terem sido empregados valores diferentes de coeficiente de troca de calor. Como foi exposto anteriormente, a transferência do calor da tira para a superfície do cilindro é muito pequena devido à sua alta velocidade de rotação. Nesse sentido, mesmo com um elevado coeficiente de troca de calor, a tira perde pouco calor para o cilindro e, portanto, não há alteração nos resultados das simulações [15].

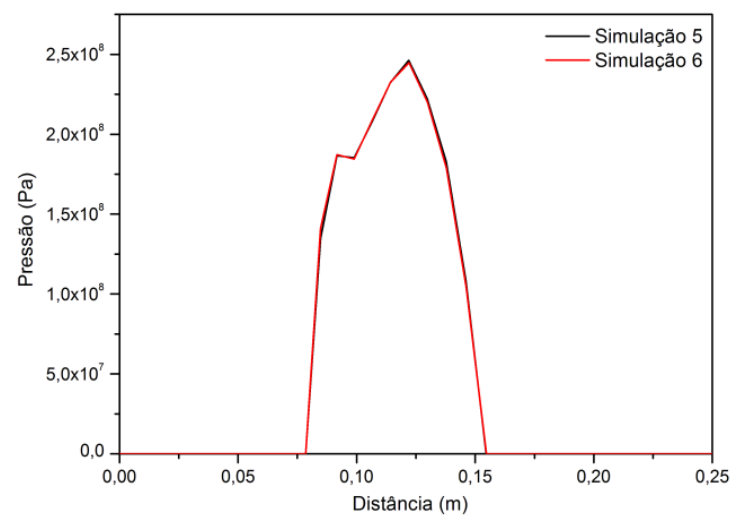

(a)

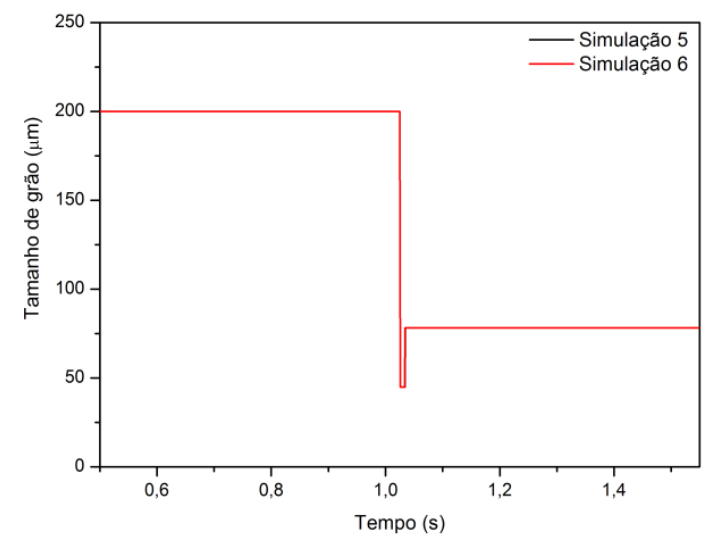

(b)

Figura 6. (a) Distribuições de pressão ao longo do arco de contato, (b) evolução do tamanho de grão austenítico [15].

\subsection{Síntese das Simulações}

\subsubsection{Força de laminação e temperatura}

A comparação entre as forças de laminação calculadas nas seis simulações pelo programa ABAQUS/Standard e a força medida nas condições industriais (CSN), para as condições apresentadas na Tabela 1, é resumida na Figura 7(a). Fica evidente que os valores de força de laminação previstos pelo ABAQUS são mais consistentes com os dados reais nas condições 5 e 6 . O erro máximo ficou abaixo de $3 \%$. Tal acurácia é satisfatória no que diz respeito aos requisitos da engenharia e, portanto, ratifica o modelamento proposto como sendo eficaz para o projeto de 


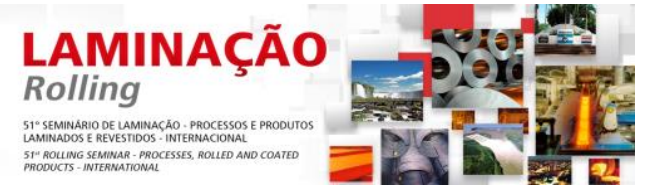

passes de laminação de tiras a quente desde que os parâmetros adequados sejam adotados no modelo [15].

O gráfico apresentado na Figura 7(b) mostra evolução da temperatura superficial de um ponto da tira desde o instante em que ela entra na região de mordida até alcançar a região entre as cadeiras 1 e 2 . Nota-se que, a taxa de resfriamento é semelhante em todos os casos. Destaca-se também que, no caso da simulação 5 , há uma queda mais acentuada de temperatura do intervalo de tempo que varia de zero até $0,04 \mathrm{~s}$. Esse é o tempo que o ponto analisado leva para passar pela zona de mordida e, como na simulação 5 empregou-se um coeficiente de troca térmica maior entre a tira e o cilindro, a queda de temperatura foi ligeiramente superior nessa condição de simulação [15].

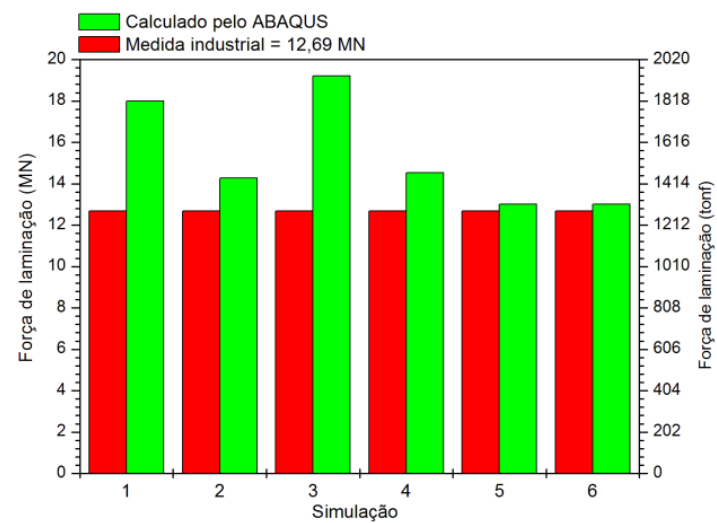

(a)

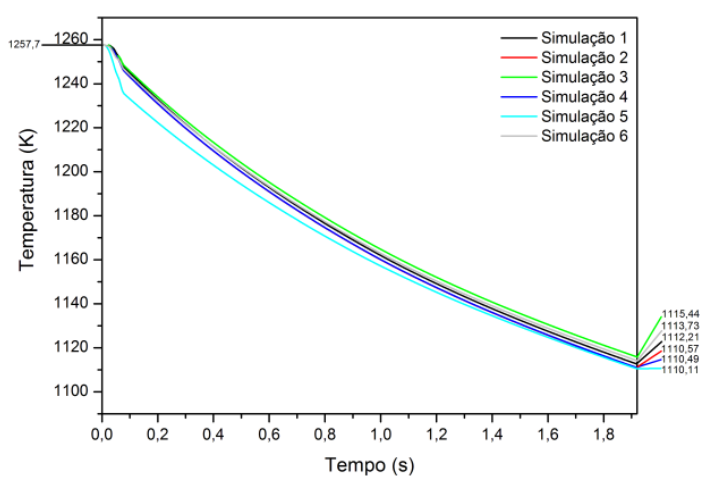

(b)

Figura 7. (a) Comparação entre as forças de laminação calculadas pelo modelo de elementos finitos proposto a medida industrial para o primeiro passe da laminação de acabamento do aço C-Mn, (b) evolução da temperatura superficial da tira[15].

A Tabela 4 mostra a comparação dos resultados de temperatura obtidos nessa dissertação, com as temperaturas em condições industriais (CSN) e, com os resultados obtidos segundo Moreira et al. [18], que empregou o método dos volumes finitos no estudo da laminação de tiras a quente, porém, sem a consideração do cilindro de trabalho. Como pode ser observado, os valores obtidos nas simulações do presente trabalho são próximos aos resultados de Moreira et al. [18].

Tabela 4. Variações de parâmetros materiais e condições de processo [15].

\begin{tabular}{|c|c|c|c|c|c|c|}
\hline $\begin{array}{c}\text { Dados } \\
\text { e } \\
\text { Simulações }\end{array}$ & $\begin{array}{c}\text { Temperatura } \\
\text { de entrada } \\
\text { (K) }\end{array}$ & \multirow{3}{*}{$\begin{array}{c}\text { Diferença: } \\
\text { CSN - } \\
\text { Simulações } \\
(\%)\end{array}$} & \multirow{3}{*}{$\begin{array}{c}\text { Diferença: } \\
\text { Moreira et al. } \\
{[18]-} \\
\text { Simulações } \\
(\%)\end{array}$} & $\begin{array}{c}\text { Temperatura } \\
\text { de saída } \\
\text { (K) }\end{array}$ & \multirow{3}{*}{$\begin{array}{c}\text { Diferença: } \\
\text { CSN - } \\
\text { Simulações } \\
(\%)\end{array}$} & \multirow{3}{*}{$\begin{array}{c}\text { Diferença: } \\
\text { Moreira et } \\
\text { al. [18] - } \\
\text { Simulaçõe } \\
\text { S } \\
(\%)\end{array}$} \\
\hline CSN & 1149,6 & & & 1164,5 & & \\
\hline $\begin{array}{c}\text { Moreira et al. } \\
\text { [18] }\end{array}$ & 1227,5 & & & 1125,6 & & \\
\hline 1 & 1257,7 & 9,40 & 2,46 & 1115,4 & 4,40 & 0,91 \\
\hline 2 & 1257,7 & 9,40 & 2,46 & 1113,7 & 4,56 & 1,07 \\
\hline 3 & 1257,7 & 9,40 & 2,46 & 1112,2 & 4,70 & 1,20 \\
\hline 4 & 1257,7 & 9,40 & 2,46 & 1110,6 & 4,85 & 1,35 \\
\hline 5 & 1257,7 & 9,40 & 2,46 & 1110,5 & 4,86 & 1,36 \\
\hline 6 & 1257,7 & 9,40 & 2,46 & 1110,1 & 4,90 & 1,40 \\
\hline
\end{tabular}

* Contribuição técnica ao $51^{\circ}$ Seminário de Laminação - Processos e Produtos Laminados e Revestidos, 28 a 31 de outubro de 2014, Foz do Iguaçu, PR, Brasil. 
7 Jupp PS. Mathematical modelling of the microestrutural evolution during the hot rolling of AA5083 aluminum alloys. A thesis submitted in partial fulfillment of the requirements for the degree of master of science, 2001.

8 Bombac D, Peet M, Zenitani S, Kimura S, Kurimura T, Bhadeshia HKDH. An integrated hot-rolling and microstructure model for dual-phase steels. Modelling and Simulation in Materials Science and Engineering, 2014; 22(4).

9 Maccagno TM, Jonas JJ, Hodgson PD. Spreadsheet modelling of grain size evolution during rod rolling. ISIJ International, 1996; 36(6): $720-728$.

10 Machado MLP. Modelo Termomicroestrutural para Determinação de Variáveis Operacionais e Metalúrgicas para Utilização em Simulação e/ou Controle na Laminação a Quente de Aços Carbono e Microligados. Tese de Doutorado, Vitória, 2005.

11 Fulvio SJr., Mathematical Modeling of the Hot Strip Rolling of Nb Microalloyed Steels. A Thesis Submitted to the Faculty of Graduate Studies and Research in Partial Fulfillment of the Requirements for the Degree of Doctor of Philosophy. Department of Mining and Metallurgical Engineering, McGill University, Montreal, Canada, 1999.

12 Stumpf W. Grain size modelling of a low carbon strip steel during hot rolling in a Compact Strip Production (CSP) plant using the Hot Charge Route. The Journal of The South Africa Institute of Mining and Metallurgy, Vol. 103, no. 10, pp 617-631, 2003.

13 Manohar PA, Kyuhwan L, Rollett AD, Youngseog Lee. Simulation of Microstructural Evolution in Rod Rolling of a Medium C-Mn Steel. Materials Science Forum, 2003; 426432: 3789-3794.

14 Gorni AA., Comparação entre as diversas abordagens para o cálculo da energia de ativação para a deformação a quente. REM - Revista Escola de Minas, pp. 16-21, 1998.

15 Barbosa GC. Simulação por Elementos Finitos do Processo de Laminação de Tiras a Quente de Aços C-Mn. Dissertação de Mestrado. Volta Redonda-RJ: Universidade Federal Fluminense; 2014.

16 Böesch PR, Martins Júnior V, Schaeffer L. Determinação do coeficiente de atrito pelo ensaio de compressão do anel: uma revisão. Revista Thema, 2011; 8(1).

17 Ebrahimi R, Shafiei E. Recrystallization: Mathematical Modeling of Single Peak Dynamic Recrystallization Flow Stress Curves in Metallic Alloys. Edited by Krzysztof Sztwiertnia, 2012.

18 Moreira LP, Castro JA, Barbosa R, Silva AJ. A finite volume model for the simulation of the strip rolling process. $18^{\text {th }}$ International Congress of Mechanical Engineering, Ouro Preto-MG, ABCM, 2005.

* Contribuição técnica ao $51^{\circ}$ Seminário de Laminação - Processos e Produtos Laminados e Revestidos, 28 a 31 de outubro de 2014, Foz do Iguaçu, PR, Brasil. 